\title{
REAL HOMOGENEOUS ALGEBRAS
}

\author{
D. Ž. DJOKOVIĆ 1
}

\begin{abstract}
Let $(A, \mu)$ be a finite dimensional real algebra (not necessarily associative) with multiplication $\mu \neq 0$. Assuming that $\operatorname{Aut}(A)$ is transitive on one-dimensional subspaces we determine all such algebras. There are up to isomorphism only four such algebras, one in each of the dimensions 1, 3,6, 7 .
\end{abstract}

Introduction. For the terminology we refer to Bourbaki [3]. All the algebras considered in this paper are assumed to be finite dimensional. Let $A$ be an algebra over a field $F, \mu: A \otimes A \rightarrow A$ its multiplication and $\operatorname{Aut}(A)$ the group of algebra automorphisms of $A$. We shall say that $A$ is homogeneous if $\operatorname{Aut}(A)$ is transitive on one-dimensional subspaces of $A$, we shall say that $A$ is extremely homogeneous if $\operatorname{Aut}(A)$ is transitive on $A \backslash\{0\}$. Kostrikin [7] has shown that if char $F \neq 2, A$ extremely homogeneous and $\mu \neq 0$, then $F$ must be a finite field. On the other hand Shult [11] has shown that if $A$ is homogeneous, $F=\mathrm{GF}(q), q>2$ and $\mu \neq 0$ then $A \cong F$. The case $F=\mathrm{GF}(2)$ has been considered by Gross [4]. Świerczkowski has shown [13] that when $F=R$ (the real field) and $A$ is a homogeneous Lie algebra with $\mu \neq 0$ then $A$ is isomorphic to the Lie algebra of skew-symmetric $3 \times 3$ real matrices. Many of these results have been improved by Mr. L. Sweet [12]. In particular, he has determined all two-dimensional homogeneous algebras and has shown that there are no nontrivial homogeneous algebras over an algebraically closed field.

In this paper we shall determine all real homogeneous algebras. If $A$ is an $F$-algebra and $B \subset A$ a subspace we define a multiplication in $B$ by choosing a vector space complement $C$ for $B$ in $A$ and putting

$$
\mu_{B}\left(b_{1} \otimes b_{2}\right)=\pi \mu_{A}\left(b_{1} \otimes b_{2}\right)
$$

where $\pi: A \rightarrow B$ is the projection with kernel $C$. We say then that $\left(B, \mu_{B}\right)$ is obtained from $\left(A, \mu_{A}\right)$ by truncation. Note that the definition of $\mu_{B}$ depends on the choice of $C$.

Received by the editors July 10, 1972 .

AMS (MOS) subject classifications (1970). Primary 17A99; Secondary 17B10, $20 \mathrm{G} 15$.

Key words and phrases. Homogeneous algebra, Lie group, Lie algebra, linear representation, symmetric and skew-symmetric tensors, weights.

1 This work was supported in part by the NRC-Grant A-5285.

(c) American Mathematical Society 1973 
We can apply this to quaternions $\boldsymbol{H}$ by choosing $B$ to be the subspace of pure quaternions and $C=R \cdot 1$. The corresponding truncated algebra $\overline{\boldsymbol{H}}$ will be called the algebra of pure quaternions; it is isomorphic to the three-dimensional Lie algebra mentioned above. Similarly, if we take $A=O$ the algebra of octonions, choose $B$ to be the subspace of pure octonions and $C=R \cdot 1$ then the corresponding truncated algebra $\bar{O}$ will be called the algebra of pure octonions. It is well known that these two algebras are homogeneous.

Let $\boldsymbol{T}=\boldsymbol{C}^{3}$ considered as a real vector space and define the multiplication in $\boldsymbol{T}$ as follows: If $x, y \in \boldsymbol{T}$ and

then

$$
x=\left(\begin{array}{c}
\xi_{1} \\
\xi_{2} \\
\xi_{3}
\end{array}\right), \quad y=\left(\begin{array}{c}
\eta_{1} \\
\eta_{2} \\
\eta_{3}
\end{array}\right),
$$

$$
x \cdot y=\left(\begin{array}{l}
\bar{\xi}_{2} \bar{\eta}_{3}-\bar{\xi}_{3} \bar{\eta}_{2} \\
\bar{\xi}_{3} \bar{\eta}_{1}-\bar{\xi}_{1} \bar{\eta}_{3} \\
\bar{\xi}_{1} \bar{\eta}_{2}-\bar{\xi}_{2} \bar{\eta}_{1}
\end{array}\right) .
$$

Then $T$ is a homogeneous algebra over reals of dimension 6 . The group SU(3) acts on $\boldsymbol{T}$ by matrix multiplication and these multiplications are automorphisms of the algebra $T$.

Result and proof. Let $(A, \mu)$ be a homogeneous $F$-algebra, $\mu \neq 0$ and $G=\operatorname{Aut}(A)$. Then $A$ is irreducible as a $G$-module. Let $\Gamma$ be any subgroup of $G$ such that $A$ is an irreducible $\Gamma$-module. The multiplication $\mu: A \otimes A \rightarrow A$ is a homomorphism of $\Gamma$-modules. If char $F=0$ then $A \otimes A$ is a semisimple $\Gamma$-module (see [5, p. 85]). Thus $A$ has to be isomorphic as a $\Gamma$-module to a direct summand of $A \otimes A$.

THEOREM. If $(A, \mu)$ is a real homogeneous algebra and $\mu \neq 0$ then it is isomorphic to $\boldsymbol{R}, \bar{H}, \boldsymbol{T}$ or $\overline{\boldsymbol{O}}$.

Proof. If $\operatorname{dim} A=n=1$ this is clear. Let $n \geqq 2$ and identify the sphere $S^{n-1}$ with the manifold of oriented one-dimensional subspaces of $A$. We have the canonical maps $A \backslash\{0\} \rightarrow S^{n-1} \rightarrow P(A)$ where $P(A)$ is the associated projective space. The action of $G$ on $A$ induces an action on $S^{n-1}$ and $P(A)$. The action of $G$ on $P(A)$ is transitive since $A$ is a homogeneous algebra. Since $G$ is a real Lie group its identity component $G_{0}$ is also transitive on $P(A)$ and $S^{n-1}$.

If $n \geqq 3$ then $S^{n-1}$ is simply connected and, by a result of Montgomery [9], $G_{0}$ has a compact subgroup $\Gamma$ which is also transitive on $S^{n-1}$. By 
taking a subgroup of $\Gamma$ (if necessary) we can assume in addition that it is a simple Lie group (see [8]). The possible groups $\Gamma$ which satisfy all these conditions have been determined by Montgomery and Samelson [8] and Borel [1] and [2]. They are as follows:

$\mathrm{SO}(n), \mathrm{SU}(m)$ for $n=2 m$,

$\mathrm{SP}(m)$ for $n=4 m$, Spin(9) for $n=16$,

$\operatorname{Spin}(7)$ for $n=8, G_{2}$ for $n=7$.

Also, the actions of these groups on $S^{n-1}$ are equivalent to the orthogonal actions obtained by embedding $\mathrm{SO}(n), \mathrm{SU}(m), \mathrm{Sp}(m)$ in $\mathrm{SO}(n)$ in the usual way; $G_{2}$ is embedded in $\mathrm{SO}(7)$ as the automorphism group of $O$ restricted to $\overline{\boldsymbol{O}}$. The embeddings of Spin(9) and $\operatorname{Spin}(7)$ are given by the real spin representations $\Delta_{9}$ and $\Delta_{7}$. These results have been proved by Poncet [10].

Since $\mathrm{SU}(m) \subset \mathrm{SO}(n)(n=2 m)$ and $\mathrm{Sp}(m) \subset \mathrm{SU}(2 m)(n=4 m)$ we can reduce the above list to the following

(i) $n$ odd, $\Gamma=\mathrm{SO}(n)$ or $G_{2}$ (if $n=7$ );

(ii) $n=2 k, k$ odd, $\Gamma=\mathrm{SU}(k)$;

(iii) $n=4 k, \Gamma=\mathrm{Sp}(k)$ or $\operatorname{Spin}(9)$ (if $n=16$ ) or $\operatorname{Spin}(7)$ (if $n=8$ ).

We shall now consider each of these possibilities.

(i) If $\Gamma=\mathrm{SO}(n)$ then $A \otimes A$ decomposes into direct sum of the symmetric and the skew-symmetric parts. The skew-symmetric part is an irreducible $\Gamma$-module. The symmetric part decomposes into two irreducible summands one of them being the trivial module. By comparison of the dimensions we see that $A$ is not isomorphic as a $\Gamma$-module to any of the summands in $A \otimes A$ if $n \geqq 5$. In the case $n=3, A$ is isomorphic to the skew-symmetric part of $A \otimes A$. Thus there exists a nonzero $\Gamma$-homomorphism $\mu: A \otimes A \rightarrow A$.

Since $A$ is absolutely irreducible as a $\Gamma$-module it follows that $\mu$ is unique up to a scalar factor. This means that the algebra $(A, \mu)$ is unique up to isomorphism. It is clear that this is the algebra of pure quaternions $\boldsymbol{H}$.

Now, assume that $\Gamma$ is the simple real Lie group $G_{2}$ and $n=7$. Consider the corresponding representation of the real Lie algebra $G_{2}$ in $A$. We shall use the notation of Jacobson [6, Chapter VII, Theorem 9] to denote its highest weight by $\lambda_{2}$. The weights of this representation are all simple and they are

$$
\lambda_{2}, \quad \lambda_{1}-\lambda_{2}, \quad-\lambda_{1}+2 \lambda_{2}, \quad \lambda_{1}-2 \lambda_{2}, \quad-\lambda_{1}+\lambda_{2}, \quad-\lambda_{1}, \quad 0 .
$$

The representation $\left(\lambda_{2}\right) \otimes\left(\lambda_{2}\right)$ decomposes into symmetric and skewsymmetric parts. An irreducible representation of highest weight $2 \lambda_{2}$ is contained in the symmetric part of $\left(\lambda_{2}\right) \otimes\left(\lambda_{2}\right)$. The dimension of $\left(2 \lambda_{2}\right)$ is 27 and the dimension of the symmetric part of $\left(\lambda_{2}\right) \otimes\left(\lambda_{2}\right)$ is 28 . Hence, the symmetric part of $\left(\lambda_{2}\right) \otimes\left(\lambda_{2}\right)$ decomposes as $\left(2 \lambda_{2}\right)+(0)$ where $(0)$ stands for the trivial irreducible representation (of dimension 1). 
Similarly, by analyzing the weights of the skew-symmetric part of $\left(\lambda_{2}\right) \otimes\left(\lambda_{2}\right)$ we find that it decomposes as $\left(\lambda_{1}\right) \oplus\left(\lambda_{2}\right)$.

Thus we have $\left(\lambda_{2}\right) \otimes\left(\lambda_{2}\right)=\left(2 \lambda_{2}\right) \oplus(0) \oplus\left(\lambda_{1}\right) \oplus\left(\lambda_{2}\right)$.

We have an analogous decomposition of $A \otimes A$ as a $\Gamma$-module. Hence there exists a nonzero $\Gamma$-homomorphism $\mu: A \otimes A \rightarrow A$. Again $A$ is absolutely irreducible as a $\Gamma$-module and consequently $\mu$ is unique up to a scalar factor. This means that the algebra $(A, \mu)$ is unique up to isomorphism. Of course, this algebra is isomorphic to $\overline{\boldsymbol{O}}$ the algebra of pure octonions.

(ii) Now let $\Gamma=\mathrm{SU}(k), n=2 k$. Then $A$ can be equipped with a complex structure so that it becomes complex $\Gamma$-module of complex dimension $k$. We shall denote this complex $\Gamma$-module by $A_{c}$. Moreover, there is a positive definite hermitian form $\langle x, y\rangle$ on $A_{C}$ which is preserved by $\Gamma$. We shall also consider $A_{C}$ as a complex module over the Lie algebra $L=\operatorname{su}(k)$ of $\mathrm{SU}(k)$. Then of course, $A_{c}$ can be considered also as a module over the complexified Lie algebra $L_{C}=\operatorname{sl}(k, C)$. The real $L$-module $A$ is obtained from $A_{C}$ by restriction of scalars.

We are interested in analyzing the $L$-module $A \otimes_{R} A$. By complexification we get the $L_{\boldsymbol{C}}$-module $\left(\boldsymbol{C} \otimes_{\boldsymbol{R}} A\right) \otimes_{\boldsymbol{C}}\left(\boldsymbol{C} \otimes_{\boldsymbol{R}} A\right)$. We have $\boldsymbol{C} \otimes_{R} A=$ $M \oplus N$, where

$$
M=\{1 \otimes x-i \otimes i x \mid x \in A\}, \quad N=\{1 \otimes x+i \otimes i x \mid x \in A\},
$$

are $L_{C}$-submodules. The map

$$
A_{C} \rightarrow M, \quad x \mapsto 1 \otimes x-i \otimes i x,
$$

is an isomorphism of $L_{C}$-modules. The bilinear form $\phi$ on $C \otimes_{R} A$ which sends $(\alpha \otimes x, \beta \otimes y) \mapsto \alpha \beta\langle x, y\rangle$ is $L_{C}$-invariant and nondegenerate. If we agree that $\langle x, y\rangle$ is linear in the first variable then the restriction of $\phi$ to $M \times N \rightarrow C$ is also nondegenerate since

$$
\phi(1 \otimes x-i \otimes i x, 1 \otimes y+i \otimes i y)=4\langle x, y\rangle .
$$

Hence the $L_{C}$-module $N$ is isomorphic to the contragredient of $M$, i.e., $C \otimes_{R} A \cong A_{C} \oplus A_{C}^{*}$. It follows that the complexification of $A \otimes_{R} A$ is isomorphic to

$$
\left(A_{C} \otimes_{C} A_{C}\right) \oplus\left(A_{C}^{*} \otimes_{C} A_{C}^{*}\right) \oplus 2\left(A_{C} \otimes_{C} A_{C}^{*}\right)
$$

as an $L_{C}$-module.

The highest weight of $A_{C}$ is $\lambda_{1}$ and that of $A_{C}^{*}$ is $\lambda_{k-1}$. Here we use again the notation of Jacobson [6, Chapter VII, Theorem 6]. By an easy computation we find that

$$
\begin{aligned}
\left(\lambda_{1}\right) \otimes\left(\lambda_{1}\right) & =\left(2 \lambda_{1}\right) \oplus\left(\lambda_{2}\right), \\
\left(\lambda_{k-1}\right) \otimes\left(\lambda_{k-1}\right) & =\left(2 \lambda_{k-1}\right) \oplus\left(\lambda_{k-2}\right), \\
\left(\lambda_{1}\right) \otimes\left(\lambda_{k-1}\right) & =\left(\lambda_{1}+\lambda_{k-1}\right) \oplus(0),
\end{aligned}
$$


where $(\lambda)$ denotes the isomorphism class of an irreducible $L_{C}$-module of highest weight $\lambda$.

If $k>3$ then we see from these decompositions that $A$ cannot be a direct summand of $A \otimes A$.

When $k=3$ we see that $A \otimes A$ contains $A$ as a direct summand with multiplicity one. Hence, there exists a nonzero $\Gamma$-homomorphism $\mu: A \otimes A \rightarrow A$ and the algebra $(A, \mu)$ is homogeneous and has dimension 6 . The endomorphism ring of the $\Gamma$-module $A$ is the complex field $\boldsymbol{C}$ and it follows easily that $(A, \mu)$ must be isomorphic to the algebra $T$ described in the introduction.

(iii) In all these cases $-I \in \Gamma$ and $(-I) \otimes(-I)$ is the identity map on $A \otimes A$. Hence $A \otimes A$ is not faithful as a $\Gamma$-module and $A$ cannot be isomorphic to a direct summand of $A \otimes A$.

It remains to consider the case $n=2$. Assume that $A \otimes A=V_{1} \oplus V_{2} \oplus V_{3}$ where $V_{1}$ is the skew-symmetric part, $V_{2} \oplus V_{3}$ the symmetric part and $V_{3} \cong A$. Let $\pi_{i}: A \otimes A \rightarrow V_{i}(i=1,2,3)$ be the associated projections. If $e_{1}, e_{2}$ is a basis of $A$ then $v_{1}=e_{1} \otimes e_{2}-e_{2} \otimes e_{1}$ spans $V_{1}$. Define the bilinear form $f: A \otimes A \rightarrow R$ by $\pi_{1}(x \otimes y)=f(x, y) v_{1}$. Then $f$ is skew-symmetric and $f(\sigma x, \sigma y)=(\operatorname{det} \sigma) f(x, y)$ for all $\sigma \in \mathrm{GL}(A)$. Similarly, define a bilinear form $g: A \otimes A \rightarrow R$ by $\pi_{2}(x \otimes y)=g(x, y) v_{2}$ where $v_{2} \in V_{2}$ is a fixed nonzero tensor. Then $g$ is symmetric and we must have

$$
g(\sigma x, \sigma y)=(\operatorname{det} \sigma)^{2} g(x, y)
$$

for $\sigma \in G_{0}$ and $x, y \in A$. The factor $(\operatorname{det} \sigma)^{2}$ is obtained by considering the determinants of the restrictions of $\sigma \otimes \sigma$ to $V_{i}$ for $i=1,2,3$. Both $f$ and $g$ are nondegenerate and we can write $g(x, y)=f(\rho x, y)$ for some fixed $\rho \in \mathrm{GL}(A)$. Then we must have

$$
f(\rho \sigma x, \sigma y)=(\operatorname{det} \sigma)^{2} f(\rho x, y), \quad f\left(\sigma^{-1} \rho \sigma \rho^{-1} x, y\right)=(\operatorname{det} \sigma) f(x, y) .
$$

Thus $\pi_{1} \circ\left(\sigma^{-1} \rho \sigma \rho^{-1} \otimes I\right)=(\operatorname{det} \sigma) \pi_{1}$ and consequently $\sigma^{-1} \rho \sigma \rho^{-1}$ must be a scalar transformation, i.e., $\sigma^{-1} \rho \sigma \rho^{-1}= \pm I$. Since $G_{0}$ is connected we have $\rho \sigma=\sigma \rho$ for all $\sigma \in G_{0}$. It follows from a formula above that det $\sigma=1$ for $\sigma \in G_{0}$. Hence $g$ is a $G_{0}$-invariant nondegenerate symmetric form. Since $G_{0}$ is transitive on $\boldsymbol{P}(A)$ we conclude that $g$ must be definite, say positive definite. By considering $A$ as a Euclidean space with $g$ as an inner product it is clear that $G_{0}=\mathrm{SO}(2)$.

But now $A \otimes A$ is not a faithful $G_{0}$-module because $-I \in \mathrm{SO}(2)$ and we have a contradiction. This completes the proof.

REMARK. We could have dismissed the case $n=2$ because it has been been shown by L. Sweet that the two-dimensional homogeneous algebras exist only over GF(2). 
I am very grateful to the editor and another referee for their critical comments on the first version of this paper.

\section{REFERENCES}

1. A. Borel, Some remarks about Lie groups transitive on spheres and tori, Bull. Amer. Math. Soc. 55 (1949), 580-587. MR 10, 680.

2. - Le plan projectif des octaves et les sphères comme espaces homogènes, C.R. Acad. Sci. Paris 230 (1950), 1378-1380. MR 11, 640.

3. N. Bourbaki, Eléments de mathématique. Algebre. Chaps. 1-3, Hermann, Paris, 1970. MR 43 \#2.

4. F. Gross, Finite automorphic algebras over GF(2), Proc. Amer. Math. Soc. 31 (1972), 10-14.

5. G. Hochschild, Introduction to affine algebraic groups, Holden-Day, San Francisco, Calif., 1971. MR 43 \#3268.

6. N. Jacobson, Lie algebras, Interscience Tracts in Pure and Appl. Math., no. 10, Interscience, New York, 1962. MR 26 \#1345.

7. A. I. Kostrikin, On homogeneous algebras, Izv. Akad. Nauk SSSR Ser. Mat. 29 (1965), 471-484; English transl., Amer. Math. Soc. Transl. (2) 66 (1968), 130-144. MR 31 \#219.

8. D. Montgomery and H. Samelson, Transformation groups on spheres, Ann. of Math. (2) 44 (1943), 454-470. MR 5, 60.

9. D. Montgomery, Simply connected homogeneous spaces, Proc. Amer. Math. Soc. 1 (1950), 467-469. MR 12, 242.

10. J. Poncet, Groupes de Lie compacts de transformations de l'espace euclidien et les sphères comme espaces homogenes, Comment. Math. Helv. 33 (1959), 109-120. MR 21 \#2708.

11. E. E. Shult, On the triviality of finite automorphic algebras, Illinois J. Math. 13 (1969), 654-659. MR 40 \#1442.

12. L. Sweet, On homogeneous algebras, Ph.D. Thesis, University of Waterloo, 1973.

13. S. Świerczkowski, Homogeneous Lie algebras, Bull. Austral. Math. Soc. 4 (1971), 349-353. MR 43 \#6277.

Department of Pure Mathematics, University of Waterloo, Waterloo, OnTaRio, Canada 